\section{DARIER'S DISEASE PRESENTING AS RECURRENT ECZEMA HERPETICUM IN 10-YEAR OLD BOY: CASE REPORT}

Marta Navratil*, Nives Pustišek, Irena Ivković-Jureković, Suzana Ožanić Bulić. Children's Hospital Zagreb

10.1136/archdischild-2021-europaediatrics.150

Eczema herpeticum $(\mathrm{EH})$ is a disseminated herpes simplex infection of the skin most commonly occurring in patients with atopic dermatitis (AD), seborrheic dermatitis, Hailey-Hailey disease, allergic contact dermatitis, psoriasis and Darier's disease (DD). It is considered a dermatologic emergency due to its high mortality rate if misdiagnosed or left untreated.

We present a case of a 10 -year-old boy with a longstanding history of $\mathrm{AD}$ referred to Allergology and Immunology Department for recurrent eczema herpeticum secondary to herpes simplex 1 (HSV-1) infection and possible primary immunodeficiency.

For the past two years, the patient has received chlorpromazine therapy for aggressive behaviour. First episode of $\mathrm{EH}$ was diagnosed at the age of six following intensive topical corticosteroid therapy for $\mathrm{AD}$ and sun exposure, another known risk factor for HSV infection. After the third $\mathrm{EH}$ episode, prophylaxis with oral acyclovir was commenced. The skin changes were treated with topical steroids and oral antibiotics in flares of the disease, with poor clinical response. On presentation he was of good general condition, adipose, of unremarkable somatic status except numerous symmetrical yellowish-brown keratotic papules and plaques over the forehead, cheeks and lateral side of the neck. The nail plate had multiple red and white longitudinal streaks. The allergy tests revealed increased total immunoglobulin $\mathrm{E}$ ( $\mathrm{IgE}$ ) and sensitization to ragweed. Immunological work up showed normal immunoglobulins and good specific immunity but decreased

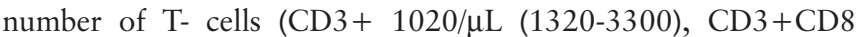
$+281 / \mu \mathrm{L}$ (390-1100) with normal $\mathrm{T}$ cell response after antigen stimulation. Skin biopsy confirmed Darier's disease. Oral retinoids were started with good clinical response.

Acyclovir prophylaxis was continued. We present the case of the 10-year-old patient with DD and impaired T-cell-mediated immunity initially misdiagnosed as AD. The occurrence of $\mathrm{EH}$ in patients with $\mathrm{DD}$ is rare and possibly related to immune system dysfunction as additional risk factor along with impaired skin barrier. This case highlights the importance of early recognition of the association of DD with impaired cellular immunity and the importance of adequate treatment due to possible fatal outcomes.

\section{CLINICAL EFFICACY OF BAC-SET ${ }^{\circledR}$ FORTE MULTISTRAIN PROBIOTICS COMPLEX IN THE PREVENTION OF ADENOTONSILLAR PATHOLOGY IN PRESCHOOL CHILDREN}

Vera Vavilova*, Alexsandr Vavilov, Asya Cherkaeva, Irina Nechaeva, Vitaliy Tiuliukin. Kemerovo State Medical University

\subsection{6/archdischild-2021-europaediatrics. 151}

Goal of research: to study Bac-Set ${ }^{\circledR}$ Forte multistrain probiotic complex efficacy and safety in humans in preschool children with adenotonsillar pathology.
Materials and Methods For the period 2016 - 2019, 346 children (3-6 years old) with a history of chronic pathology of pharyngeal and palatine tonsil and suffering from frequent recurrent respiratory infections were observed.

The average age was $4.53 \pm 2.71$ years. Preschool children of the treatment group $(n=230)$ received multistrain probiotic complex Bac-Set ${ }^{\circledR}$ Forte as a prevention of exacerbations of chronic nasopharyngeal pathology on a daily basis, in addition to irrigation and elimination therapy (nasal shower with $0.9 \%$ $\mathrm{NaCl}$ solution); 1 capsule a day was prescribed for 30 days. The control group $(n=116)$ was on irrigation and elimination therapy only. The analysis of the efficiency and safety of the multistrain probiotic complex was carried out before and after the preventive course.

Results a year before the start of the preventive course with Bac-Set ${ }^{\circledR}$ Forte multistrain probiotic complex, the pharyngeal tonsil hypertrophy of degree 2 and with complication by adenoiditis was observed in $76.3 \%$ of children in the treatment group and in $75.8 \%$ of children in the control group $(p=0.2376)$. By the end of the study, only $30.4 \%$ of patients in the treatment group showed no improvement in the clinical picture $(p=0.000)$. A year after the recovery stage, $62.7 \%$ of patients which were on the multistrain probiotic complex had recovered nasal breathing $(p=0.001)$; the symptoms of adenoiditis were almost stopped in $51.8 \%$ of patients $(p=0.000)$; $82.7 \%$ of patients had a decrease in the volume of the pharyngeal tonsil from degree 2 to $1 \quad(p=0.000)$; $78.9 \%$ of patients had a normalization of the rhinoscopic picture $(p=0.000)$; endoscopic control confirmed a decrease in the size of the palatine tonsils in $56.8 \%$ of patients $(p=0.000)$. The degree of hypertrophy of the pharyngeal and palatine tonsils in preschool children who did not receive the multistrain probiotic complex did not change and even increased in dynamics in $81.4 \%$ of patients.

Conclusions The study results have confirmed the efficiency and high tolerability of Bac-Set ${ }^{\circledR}$ Forte multistrain probiotic complex. Prospective observation of children who was on BacSet ${ }^{\circledR}$ as a prevention of exacerbations of adenotonsillar pathology have confirmed its efficacy in the formation of respiratory tract immunity. The obtained data allow us to recommend the addition of Bac-Set ${ }^{\circledR}$ Forte multistrain probiotic complex in programs for the prevention of exacerbations of adenotonsillar pathology in children.

\section{ANAPHYLAXIS TO COLD TRIGGERED BY CAMPYLOBACTER JEJUNI ENTERAL INFECTION: A CASE REPORT}

Srðan Banac*, Iva Volarić. Department of Paediatrics, Clinical Hospital Centre Rijeka, Croatia - Department of Paediatrics, Faculty of Medicine, University Rijeka, Croatia

\subsection{6/archdischild-2021-europaediatrics. 152}

Background Anaphylaxis in children may sometimes be difficult to recognise and needs a high degree of suspicion. The most common causes are food allergens, medications and hymenoptera venoms. Physical factors like extensive cold contact can also result in systemic reaction ranging from generalized urticaria to anaphylaxis. Case report: A 12-year old boy was getting into the cold sea and he suddenly developed general malaise, nausea, abdominal pain, diarrhea and itchy skin. When emergency medical assistance arrived his blood pressure was $70 / 50 \mathrm{mmHg}$ and he was noted to have generalized hives 